\title{
The association between excess weight and body composition measurements in a pediatric population
}

\section{Eirini Kostopoulou ( $\nabla$ irekost@upatras.gr)}

University General Hospital of Patras https://orcid.org/0000-0002-7051-7537

\section{Aikaterini Avgeri}

University General Hospital of Patras: Panepistemiako Geniko Nosokomeio Patron Panagia e Boetheia

Spyridon Skiadopoulos

University of Patras School of Health Sciences: Panepistemio Patron Schole Epistemon Ygeias

\section{Gabriel Dimitriou}

University General Hospital of Patras: Panepistemiako Geniko Nosokomeio Patron Panagia e Boetheia loannis Giannakopoulos

University General Hospital of Patras: Panepistemiako Geniko Nosokomeio Patron Panagia e Boetheia

\section{Research}

Keywords: overweight, pediatric obesity, body composition, fat mass, skeletal muscle mass

Posted Date: March 30th, 2021

DOI: https://doi.org/10.21203/rs.3.rs-334558/v1

License: (c) (i) This work is licensed under a Creative Commons Attribution 4.0 International License.

Read Full License 


\section{Abstract}

Background: The prevalence of childhood obesity in Greece is among the highest in Europe. Body composition may be used as a screening tool to identify children with excess weight and those at risk for developing metabolic complications. The aim of the study was to provide data on body composition indices from a large Greek pediatric population and investigate the possible association between these indices and obesity.

Methods: 1453 schoolchildren, $51.2 \%$ males, aged 10-12 years old, were recruited from the third in size city in Greece. Anthropometric and body composition indices (weight, height, body mass index (BMI), fat mass (FM), FM\%, fat mass index (FMI), skeletal muscle mass (SMM), SMM\%, skeletal muscle mass index (SMI) and muscle-to-fat ratio (MFR)), were assessed.

Results: i) The rates of overweight and obesity were $25.9 \%$ (Males: $24.4 \%$, females: $27.5 \%$ ) and $12.6 \%$ (males: $13.4 \%$, females: $11.7 \%$ ), respectively. ii) Males had significantly higher SMM, SMM\% and MFR, and lower FM\% compared to females. iii) No differences were observed in body composition between younger (10-11-year-old) and older (11-12-year-old) children. iv) Higher FM, FM\%, FMI, SMM, SMI and lower SMM\% and MFR values were found in children with overweight or obesity.

Conclusion: A direct association between excess weight and body composition measurements, such as $\mathrm{FM}, \mathrm{FM} \%$, FMI, SMM, SMM\%, SMI and MFR, was found in a large pediatric school-population. These body composition analysis measurements may potentially serve as a useful screening-tool with both research and clinical applications in assessing obesity, but also for predicting obesity-related complications.

\section{Background}

Childhood obesity is currently recognized as a global epidemic due to its worryingly rising prevalence (1). Whereas obesity in adults has been associated with increased morbidity due to a heightened risk of cardiovascular diseases, metabolic dysregulation and musculoskeletal disorders (2), such an association is less prominent in the case of children and adolescents. The prevalence of childhood obesity in Greece is among the highest in Europe $(3,4)$.

There is increasing interest in the study and use of body composition as a screening tool for the identification of children with excess weight and those at risk for the development of potential obesityrelated health consequences. Choosing the appropriate method of body composition analysis is challenging knowing the changes in body composition that occurs during growth.

There is no in vivo gold-standard for the measurement of body composition in children. More than one methods may be appropriate for use based on the information that is desired. Weight for length is commonly used for infants (5). Waist circumference is another anthropometric measure used to estimate overall and visceral adiposity and has been associated with increased cardiometabolic risk in childhood (6). Upper arm fat and muscle areas expressed as upper arm circumference and triceps skin fold 
thickness have been used as indices of nutritional status, but these parameters vary with age and body size which restricts their accuracy (7).

The body mass index (BMI), calculated as weight $(\mathrm{kg}) /$ stature $(\mathrm{m})^{2}$, represents the most widely used index in children above the age of 2 years and in adults. Reference values have been established (8). Overweight is defined in children and adolescents as a BMI between the $85^{\text {th }}$ and $95^{\text {th }}$ percentile for age and sex and obesity as a BMI of above the $95^{\text {th }}$ percentile for age and sex (9). BMI is used to define obesity, but it does not take into consideration body fat or muscle composition. As a result, children with normal weight or BMI may have excess adiposity, hence be at increased risk for the development of cardiometabolic disease (10). Due to the limitations of BMI use with regard to metabolic risk classification and generalizability across racial groups, other indices of body composition are also considered in order to serve as a screening tool for identifying cardiometabolic disease.

Total body fat mass (FM) levels and distribution have been proposed as stronger determinants of cardiometabolic risk compared to BMI (11). Also, total trunk and visceral fat mass levels are positively associated with hypertension in young children (12). Percent body fat (FM\%) \{fat mass (kg) / body mass $(\mathrm{kg}) * 100\}$ can be obtained from body composition methods that estimate fat mass and differentiates between fat mass and free fat mass. It is more useful compared to BMI (13) and can discriminate the presence or absence of metabolic syndrome in children and adolescents with moderate accuracy (14). Laurson et al have determined the 85th percentile for males and the 68th percentile for girls as the optimal cut-offs for percent body fat for the identification of cardiometabolic risk, however, there are no internationally proposed cut-off values for total body fat mass and percent fat mass (15).

In addition, skeletal muscle mass (SMM) is an essential component of body composition that reflects the nutritional status of children (15) and is considered as an independent marker of metabolic health and physical performance and a major site of insulin mediated glucose disposal, playing a key role in glucose homeostasis (16). Muscular strength is positively correlated with insulin sensitivity in children and adolescents, whereas low muscle mass is associated with increased metabolic risk (17). SMM is the most abundant sensitive tissue and accounts for $85 \%$ of all insulin-mediated glucose utilization (18). SMM and fat mass have opposing effects on insulin sensitivity and energy disposal. Reduced SMM may co-exist and act synergistically with increased body fat mass, which is also associated with insulin resistance, thus low skeletal muscle-to-body fat ratio (MFR) could be a predictor for future development of metabolic disease and osteoporosis (19). The balance between FM and SMM may predict in a more accurate manner the risk for metabolic disease compared to the BMI, nonetheless this theory remains to be confirmed.

Among the different methods of evaluating muscle mass, the DXA is considered as the most accurate/gold-standard for assessing three components of body composition: muscle mass, fat mass and bone mass in children (20). Despite its significant advantages, the high cost of the exam, the necessity for trained operators and the need for specialized infrastructure, limits the usefulness of the exam. On the other hand, bioelectrical impedance (BIA) stands out as a simple, rapid, portable, non- 
invasive and inexpensive method for assessing body composition and is able to distinguish between fat mass (FM) and free fat mass (FFM). Of note, the use of SMM as a surveillance tool is limited because of the absence of a reference range adjusted for age for children and adolescents (21).

The fat mass index (FMI: fat mass $(\mathrm{kg})$ / stature $(\mathrm{m})^{2}$ ) and skeletal muscle mass index (SMI: skeletal muscle mass $(\mathrm{kg}) /$ stature $(\mathrm{m})^{2}$ have been proposed as more accurate indices of adiposity and malnutrition than BMI (22). Studies in children have shown that increases in BMI during childhood are mainly driven by increases in skeletal muscle mass index and not fat mass index, suggesting that BMI may not accurately represent adiposity in all situations (22). However, the use of FMI and SMI in children is limited due to lack of robust reference data. Further studies are required so that the best index that can serve as a screening tool is determined.

The aim of the present study was: i) to assess body composition and to record the prevalence of overweight and obesity in a pediatric population from Western Greece aged 10 to 12 years old, ii) to investigate the possible association between overweight/obesity and anthropometric indices, such as FM, $\mathrm{FM} \%, \mathrm{FMI}, \mathrm{SMM}, \mathrm{SMM} \%$, SMI, MFR and iii) to study the possible role of body composition indices in predicting future cardiometabolic complications related to obesity.

\section{Patients And Methods}

A total of 1453 children, aged 10-12 years old, were recruited from 49 elementary schools of Patras, the third in size city in Greece, during the 2014-2015 school years. These children represent almost the total of this regional population of children at the specific studied ages. Children were divided into two age ranges: 10-11 and 11-12 years old, in which they were almost equally distributed; 716 of the participants (49.3\%) were between 10 and 11 years old and the remaining 737 patients (50.7\%) were between 11 and 12 years old. Among the children, 745 were male and $94 \%$ were Greeks.

Demographic information of students, such as gender, date of birth and school class, was obtained from each school headmaster. Anthropometric and body composition measurements were obtained on school premises by two trained investigators. Several anthropometric and body composition indices were calculated and analyzed to identify those variables most strongly associated with overweight and obesity. The tested variables were: weight, height, BMI, fat mass (FM), percent fat mass (FM\%), fat mass index (FMI), skeletal muscle mass (SMM), percent skeletal muscle mass (SMM\%), skeletal muscle mass index (SMI), muscle-to-fat ratio (MFR). The different parameters were measured using non-invasive methods.

All measurements were repeated and the average value was calculated. A standardized procedure of measurements was followed in order to minimize the inter-rate variability among schools. Data collection was completed in one working day for each school.

Children's weight and height were measured in the morning. Height was measured to the nearest $0.1 \mathrm{~cm}$ with a portable stadiometer (Soehnle Stadiometer 5003) with children standing in bare feet. Weight and body composition results (fat mass, free fat mass and predicted skeletal muscle mass) were provided 
from a bioimpedance segmental body composition analyser (InBody 230). BMI was calculated as weight

divided by height squared $\left(\mathrm{kg} / \mathrm{m}^{2}\right)$. Normal weight, overweight and children and children with obesity were classified using the International Obesity Taskforce age- and gender-specific BMI cut-off criteria (23). The BMI level was categorized into two groups; the normal weight group $\left(B M \mid<85^{\text {th }}\right.$ percentile for age and sex $)$ and the excess weight group (overweight: $85^{\text {th }}-95^{\text {th }}$ percentile, obesity: $>95^{\text {th }}$ percentile).

Percent body fat (FM\%), percent skeletal muscle mass (SMM\%), FMI and SMI were calculated as follows:

FM\%: fat mass $(\mathrm{kg}) /$ body mass $(\mathrm{kg}) * 100$

SMM\%: SMM $(\mathrm{kg}) /$ body mass $(\mathrm{kg}) * 100$

Fat mass index (FMI): fat mass $(\mathrm{kg}) /$ stature $(\mathrm{m})^{2}$

Skeletal muscle mass index (SMI): skeletal muscle mass $(\mathrm{kg}) /$ stature $(\mathrm{m})^{2}$

Muscle-to-fat ratio (MFR) was derived by dividing SMM (kg) by FM (kg).

The study was conducted by the University of Patras School of Medicine in collaboration with the Regional Directorate of Primary Education of Western Greece. The study protocol was approved by the Research Ethics Committee of the University Hospital of Patras in Greece (IRB number: 55344/19.12.17) and was in accordance with the Helsinki Declaration as revised in 2013. Written informed consent was obtained from the parents of the students and archived and informed assent was obtained from the students. Only children for whom parental consent was obtained were measured and included in this study.

\section{Statistical Analysis}

Statistical analysis was performed using the SPSS Statistical Software Package (IBM SPSS Statistics, version 24, Chicago, IL, USA). Specifically, descriptive statistics (mean and standard deviation) were calculated for each anthropometric and body composition measurement (i.e., weight, height, BMI, FM, FM\%, FMI, SMM, SMM\%, SMI and MFR) in: (i) Whole sample ( $n=1453)$, (ii) Males $(n=745)$ and Females $(n=708)$, (iii) Younger (10-11 years old, $n=716)$ and Older (11-12 years old, $n=737)$ children, (iv) Younger males ( $n=371)$ and Younger females ( $n=345)$, (iv) Older males ( $n=374)$ and Older females ( $n=363)$, (vi) Younger males and Older males, (vii) Younger females and Older females.

To study statistically significant differences between (i) Males and Females, (ii) Younger and Older children, (iii) Younger males and Younger females, (iv) Older males and Older females, (v) Younger males and Older males, (vi) Younger females and Older females, for each anthropometric and body composition measurement (i.e., weight, height, BMI, FM, FM\%, FMI, SMM, SMM\%, SMI and MFR), an appropriate statistical test was performed for unpaired data (Student's t-test), whereas for their distributions in BMI\% categories (i.e., $<85,85-95$ and $>95$ ) Pearson chi-square test was utilized. 
To study statistically significant differences among BMI\% categories (i.e., <85, 85-95 and >95) for each body composition measurement (i.e., FM, FM\%, FMI, SMM, SMM\%, SMI and MFR), one-way analysis of variance (ANOVA) was applied. Bonferroni adjustment was performed for multiple comparisons of BMI\% categories (i.e. $<85$ vs. $85-95,<85$ vs. $>95$ and $85-95$ vs. $>95$ ). An appropriate parametric statistical test was performed for unpaired data (Student's t-test). In all cases, the level of statistical significance was set to $\mathrm{a}=0.05$.

\section{Results}

Among the total population, 893 children (61.5\%) were of normal weight, 377 children (25.9\%) were overweight and 183 children (12.6\%) had obesity (Figure 1a). $24.4 \%$ of males were overweight vs $27.5 \%$ of females, whereas obesity was observed in $13.4 \%$ of males and $11.7 \%$ of females (Figure $1 \mathrm{~b}$ ). Prevalence of normal weight, overweight and obesity by age and gender is incorporated in Figure 1a-c.

Table 1 summarizes the sex-specific and age specific values for anthropometric and body composition measurements of the studied population.

\section{Comparison according to gender (between males and females)}

No statistically significant difference was observed between males (boys) and females (girls) with respect to weight ( $p=0.941)$, height $(p=0.121), B M I(p=0.265)$ (Table 1) and BMI\% (Pearson chi-square test, $p=0.316$ ) (Figure 1b). Also, no statistically significant difference was found between males and females with respect to FM $(p=0.052)$ and $F M I(p=0.074)$, however females had significantly higher $F M \%$ compared to males $(p<0.001)$ (Table 1$)$.

Statistically significant difference exists between males and females with respect to SMM ( $p=0.001)$, SMM\% $(p<0.001)$, SMI $(p<0.001)$ and MFR $(p<0.001)$, with boys exhibiting higher values than girls (Table 1).

\section{Comparison according to age}

No statistical difference was observed between children of the two age groups (younger children: 10-11 years old and older children: 11-12 years old) with respect to BMI\% (Pearson chi-square test, $p=0.878$ ) (Figure 1c). Specifically, 443/716 children (61.9\%) of the younger and $450 / 737(61.1 \%)$ of the older children were normal-weight, 186 of the younger children (26.0\%) and 191/737 (25.9\%) of the older children were overweight and $87 / 716(12.2 \%)$ of the younger children and $96 / 737$ (13.0\%) of the older children had obesity (Figure 1c).

Statistically significant difference was found between the younger and older children with respect to: i) height ( $p<0.001)$, ii) weight ( $<<0.001)$, iii) BMI ( $<<0.001)$, iv) SMM ( $<<0.001), v)$ FM $(p<0.001)$, and vi) SMI $(p<0.001)$. All the parameters had higher values in the older children (Table 1). On the contrary, no statistically significant difference was found between the two age groups with respect to $\mathrm{SMM} \%, \mathrm{FM} \%$, FMI, MFR (Table 1). 


\section{Younger children}

Statistically significant difference was found in FM ( $p=0.002)$, FM\% ( $p<0.001)$, FMI $(p=0.003)$, SMM $(p=0.016), S M M \%(p<0.001), S M I(p<0.001)$, and MFR $(p<0.001)$ between younger boys and younger girls (Table 2). Skeletal muscle mass indices were higher in boys and fat mass indices were higher in girls.

No statistically significant difference was found in height $(p=0.446)$, weight $(p=0.212)$ and BMI $(p=0.414)$ between younger boys and girls (Table 2).

Furthermore, no statistically significant difference exists in children aged 10-11 years old between males and females with respect to BMI\% (Pearson chi-square test, $p=0.209$ ) (Figure 1d).

\section{Older children}

Statistically significant difference was found in BMI $(p=0.020)$, SMM $(p=0.018)$, SMI $(p<0.001)$ and MFR $(p=0.029)$ between boys and girls of older children (11-12 years old). All the parameters were higher in the boys (Table 2).

No statistically significant difference between boys and girls was observed with respect to height $(p=0.158)$, weight $(p=0.219)$, SMM\% ( $p=0.094), F M(p=0.879), F M \%(p=0.261)$ and FMI ( $p=0.673)$ (Table 2). Also, no statistically significant difference exists in children aged 11-12 years old between males and females with respect to BMI\% (Pearson chi-square test, $p=0.127$ ) (Figure 1e).

\section{Comparison between younger and older male children}

Statistically significant difference was found in height ( $p<0.001)$, weight $(p<0.001), B M I(p<0.001)$, SMM $(p<0.001), F M(p<0.001), S M I(p<0.001)$ and FMI $(p=0.008)$, between younger and older male children. All the parameters were higher in the older children (Table 3). No statistically significant difference was found in SMM\%, FM\% and MFR between younger and older male children (Table 3).

Also, no statistically significant difference exists in male children $(n=745)$ between $10-11$ years old and 1112 years old with respect to BMI\% (Pearson chi-square test, $p=0.172>0.05$ ) (Figure 1f).

\section{Comparison between younger and older female children}

Statistically significant difference was observed between younger and older female children in height $(p<0.001)$, weight $(p<0.001)$, SMM $(p<0.001)$, SMM $\%(p<0.001)$, SMI $(p<0.001)$. All parameters were higher in older females (Table 3).

No statistically significant difference was found between the two age groups of the females with respect to BMI ( $p=0.267), F M(p=0.178), F M \%(p=0.216), F M I(p=0.515)$ and MFR $(p=0.276)$ (Table 3$)$.

Also, no statistically significant difference exists in female children between 10-11 years old and 11-12 years old with respect to BMI\% ( $p=0.425)$ (Pearson chi-square test, Figure 1g). 
Furthermore, pair-wise comparisons of BMI\% (i.e., $<85$ vs. $85-95,<85$ vs. $>95$ and $85-95$ vs. >95) for all the studied indexes revealed higher FM, FM\%, FMI, SMM, SMI values and lower SMM\% and MFR values in overweight children $(85<\mathrm{BMI} \%<95)$ when compared with normal weight children $(\mathrm{BMI} \%<85)$ and in children with obesity $(\mathrm{BMI} \%>95)$ when compared with overweight children $(85<\mathrm{BMI} \%<95)$ (Table 4). The same results were found when the total population was divided in males and females or when the population was divided in younger and older children (Table 4).

\section{Discussion}

The aim of the present study was to record overweight and obesity rates in a large Greek pediatric population, to identify anthropometric and body composition indices most strongly associated with excess weight and obesity-related co-morbidities and to quantify the impact of obesity on body composition. The data used in this study was drawn from schoolchildren from Patras, Western Greece.

Body composition analysis represents a useful tool with both research and clinical applications particularly in assessing obesity, as reduced muscle mass may be obscured by increased adipose tissue. The majority of the studied children were of normal body weight (61.5\%). The first major finding of the present epidemiologic study indicates that the overall prevalence of overweight and obesity in the total population were $25.9 \%$ and $12.6 \%$, respectively, which is higher than this described in other Greek pediatric populations. Specifically, a study from 40,769 Greek adolescents, aged 12-19 years, which was conducted between 2010 and 2012, showed that $23.6 \%$ were overweight and $7.5 \%$ had obesity (24).

Also, in our study, the prevalence of overweight in males was $24.4 \%$ (younger: $23.7 \%$, older: $25.1 \%$ ) and in females it was $27.5 \%$ (younger: $28.4 \%$, older: $26.7 \%$ ) and the prevalence of obesity in males was $13.4 \%$ (younger: $11.3 \%$, older: 15.5\%) and in females 11.7\% (younger: 13\%, older: 10.5\%). Hence, the overall prevalence of overweight was higher in girls compared to boys, whereas the prevalence of obesity was higher in boys than in girls. In addition, in the previously mentioned study by Grammatikopoulou et al (24), boys had a predominance compared to girls in both overweight and obesity (overweight: $27.9 \%$ vs $19.4 \%$, obesity: $8.9 \%$ vs $6.0 \%$ ). Furthermore, the rate of overweight in the boys was lower in our study compared to that of the boys of the study by Grammatikopoulou et al, but in our study the girls had significantly higher rates of overweight (24). With regard to obesity rates, these were higher in the boys and girls of our study compared to the boys and girls studied in 2010-2012 (25). In another school-based survey by Tambalis et al, polled in 2015 on 336,014 children, among children aged 10-12 years, $25.2-25.8 \%$ of the boys and $22.4-$ $25.0 \%$ of the girls were overweight and $8.2-9.0 \%$ of the boys $5.2-7.8 \%$ of the girls had obesity (25). Again the overall rate of overweight in females was higher in our study, as well as the obesity rates in both the boys and the girls. Finally, an analysis of childhood obesity in Greece during 2000-2010 documented a slightly lower prevalence of overweight among children ( $24.1 \%$ and $23.2 \%$ for boys and girls, respectively) and a lower prevalence of obesity (11.0\% and 9.2\% for boys and girls, respectively) (26). Data from all these studies suggest that excess weight, particularly in the form of obesity, has risen significantly in both male and female Greek children aged 10-12 years during the past 15 years. This propensity in increasing obesity rates suggests an obesogenic life-style, which may be explained by the economic crisis in the 
absence of more nutritious food choices or could be an overall trend. Our study population consists of a large sample of children from Western Greece, but since the sample comes from the $3^{\text {rd }}$ in size city in Greece and since primary education is compulsory in Greece, we hypothesize that it represents to a significant degree the most current obesity trend in the country as a whole.

Furthermore, males had increased skeletal muscle and the relative indices (SMM, SMM\%, SMI, MFR) compared to females and lower FM\% (Table 1), even though there was no difference in the BMI\% between the two genders. When the age was considered, the same was noted only at the younger age. SMM, SMM\%, SMI and MFR were again higher in the younger boys compared to the younger girls, whereas FM, $\mathrm{FM} \%$ and FMI were lower (Table 2), whereas no statistically significant difference was found in any of the above parameters when older boys were compared with older girls. This suggests that the difference in body composition seen between boys and girls of 10-11 years of age is not present at the age of 11-12 years old. This may be because of the altered body composition observed during transition to puberty, since girls enter puberty earlier than boys and the age of 10-11 years old is considered as pre-puberty in girls. The observation that the difference in body composition between boys and girls is restored in girls aged 11-12 years, possibly suggests that the body composition alterations in younger girls are only transient and do not place them at risk for metabolic disturbances or future metabolic complications compared to the boys.

Another interesting finding of our study is that no statistically significant difference was found between the two age groups with respect to SMM\%, FM\%, FMI, MFR (Table 1). Anthropometric measurements (weight, height, BMI, FM, SMM), were higher in older children, as expected, however body composition did not differ between the two age groups. The latter may be due to the small age difference, by only 1 year, between the two age groups.

Furthermore, the mean BMI\% was the same between younger and older children, as well as between younger and older males and younger and older females. That means that the age difference of one year did not affect the overall rate of overweight or obesity in the studied boys and girls. The same was seen with regard to $\mathrm{SMM} \%, \mathrm{FM} \%$ and MFR, suggesting that the body composition was not different in the two age groups of the children nor was it different in the two age groups of the boys. In females, although $\mathrm{FM} \%$ was similar between the two age groups, SMM\% was higher in the older girls. Hypothetically, this could be an adjustment to counteract for the increased FM\% developed in girls during the pre-pubertal period. It is well known that free fat mass decreases in girls particularly before puberty, a change that may contribute to the initiation of puberty (27).

According to the percentage body fat curves for children and adolescents in the US population, based on NHANES data from 1999 to 2004, boys aged 10-12 years old had approximately 27 percentage of body fat ( $50^{\text {th }}$ percentile) and girls had approximately 32 percentage of body fat ( $50^{\text {th }}$ percentile) $(28)$, which are both higher than the mean values of FM\% seen in our population (boys: $24.2 \pm 10.3$, girls: $26.0 \pm 9.4$ ) (Table 1). In contrast, results from children and adolescents from England and Turkey published in 2006 and 2010 , respectively, have shown a percentage body fat of approximately 17.5 ( $50^{\text {th }}$ percentile) (England) 
and a mean value of 18.5-21.4 (Turkey) in 10-12-year-old boys and 23 ( $50^{\text {th }}$ percentile) (England) and a mean value of 23.6-24.9 (Turkey) in 10-12 year old girls. The results from both countries were much lower than the results found in our study, suggesting that Greek children of the above age have significantly higher excess fat. However, it should be noted that the data from the US, England and Turkey were not obtained during the same time periods, but years before our data was collected.

A recent study by Kim et al conducted in children from Korea between 2009 and 2011, showed that height, weight, SMM, SMM\% and SMI were lower in Korean boys and girls aged 10-12 years compared to our population. This finding is important, because it has been shown that elevated values of SMM may increase sensitivity to insulin (29). On the other hand, low SMM values relate to metabolic risk factors and resistance to insulin (17), including the risk for osteoporosis. With regard to MFR, although no international reference range has been established, comparison with MFR data from the same Korean pediatric population (30) shows that MFR is higher in our population in both the males and females (mean \pm SD: Greek boys: $2.20 \pm 1.62$ vs Korean boys: $1.2 \pm 0.1$, Greek girls: $1.86 \pm 1.39$ vs Korean Girls: $1 \pm 0.1$ ), possibly suggesting a better metabolic profile. Interestingly, the mean MFR in normal weight Korean children aged 10-12 years ranged from $1.36 \pm 0.44$ to $1.30 \pm 0.5$ for boys and from $1.02 \pm 0.27$ to $1.09 \pm 0.31$ for girls. Mean MFR values in our population was $2.82 \pm 0.07$ for boys and $2.24 \pm 0.06$ for girls, suggesting a better metabolic profile. In Korean children with overweight or obesity, MFR ranged between $0.68 \pm 0.13$ to $0.71 \pm 0.16$ for boys and from $0.70 \pm 0.10$ to $0.71 \pm 0.12$ for girls, as opposed to our population with overweight or obesity where MFR ranged from $1.33 \pm 0.10$ to $0.90 \pm 0.14$ respectively, for boys and from $1.43 \pm 0.09$ to $0.92 \pm 0.14$ for girls, respectively, suggesting that even children with excess weight from Western Greece had a more favourable body composition compared to Korean age-matched children. Of course, possible racial or ethnic differences in body composition should not be ignored.

An additional very interesting finding of our study is that overweight and obesity in the studied population were positively correlated with higher FM, FM\%, FMI, SMM and SMI and negatively correlated with SMM\% and MFR (higher FM, FM\%, FMI, SMM, SMI and lower SMM\% and MFR in overweight children vs normal weight children and in children with obesity vs overweight children). The same results were found when the population was divided into boys and girls or younger and older children (Table 3). This finding suggests a direct association between the studied indices and excess weight and also highlights the potential significance of these indices as predictive markers for potential metabolic disturbances and future cardiovascular complications. Knowing that skeletal muscle mass represents an independent marker of metabolic health and insulin sensitivity (17), and that total body fat mass is stronger as a determinant of cardiometabolic risk compared to BMI (19) and that fat mass levels are positively associated with hypertension (12), the finding that measures of body composition can directly been associated with excess body weight is of major importance, as they may also serve as predictive markers of obesity-related co-morbidities.

Strengths of the present study include that it is a large-scale epidemiological study conducted in a homogeneous sample of children. A standard protocol was used for the measurement of anthropometric data and there was no selection bias. Because of the large sample size, statistical significance could

Page 10/20 
easily be achieved. However, the study is not nationally representative and not representative of all age groups, as it included only $5^{\text {th }}$ and $6^{\text {th }}$ graders, which does not allow for generalization of the present findings. Thus, the findings of our study should be interpreted cautiously. Additional limitations of the present study include that no dietary intake assessment was performed and physical activity levels were not recorded. Also, our study did not take into account the contribution of puberty to fat mass, because pubertal staging was not performed, as it was practically difficult in the school setting.

In conclusion, our results offer, to a significant degree, an update of data on the most recent overweight and obesity prevalence trends in children in Greece and add to the existing knowledge that overweight and obesity rates in Greek children are alarmingly elevated. Such findings necessitate the development of effective public health interventions from public health policy makers in order to prevent further increase in overweight and obesity rates and to address this health problem and the related co-morbidities. Such interventions include adherence to the Mediterranean diet, increasing physical activity and reducing screen time. Childhood is an advantageous period to apply effective prevention strategies for obesity, before dietary habits are fully established.

To our knowledge, this is the first study to examine the association between pediatric overweight and obesity and a variety of anthropometric and body composition measurements in a pediatric population and to suggest that these measurements are directly associated with excess weight and could potentially serve as screening tools for predicting obesity-related complications. To the present, there are no established cut-off values for anthropometric and body composition indices above which complications can be best predicted in children. Further studies are needed to confirm these findings and establish widely acceptable cut-off values for identifying children at high risk of cardiometabolic syndrome.

\section{List Of Abbreviations}


BMl: Body mass index

FM: Fat mass

SMM: Skeletal muscle mass

MFR: Skeletal muscle-to-body fat ratio

MRI: Magnetic resonance imaging

CT: Computerized tomography

DXA: Dual X-ray Absorptiometry scan

BIA: Bioelectrical impedance

FFM: Free fat mass

FMl: Fat mass index

SMI: Skeletal muscle mass index

MFR: Muscle-to-fat ratio

\section{Declarations}

Funding: This research did not receive any specific grant from funding agencies in the public, commercial, or not-for-profit sectors.

Competing interests statement: The authors declare that they have no conflict of interest.

Ethics approval and consent to participate: The study was approved by the Research Ethics Committee of the University Hospital of Patras in Greece (IRB number: 55344/19.12.17) and was in accordance with the Helsinki Declaration as revised in 2013. Written informed consent was obtained from the parents of the students and archived and informed assent was obtained from the students.

Consent for publication: Not applicable

Availability of data and materials: The datasets used and/or analysed during the current study are available from the corresponding author on reasonable request.

Authors' contributions: EK was responsible for data analysis and interpretation, writing of the first draft and has approved the submitted version. AA was responsible for data acquisition and analysis, writing of the first draft and has approved the submitted version. SS participated in data analysis and interpretation, statistical analysis and has approved the submitted version. GD was responsible for the conception of the study, data interpretation and has approved the submitted version. IG was responsible for the design of the study, data acquisition and analysis, and has approved the submitted version. All authors have agreed both to be personally accountable for the author's own contributions and to ensure that questions related 
to the accuracy or integrity of any part of the work, even ones in which the author was not personally involved, are appropriately investigated, resolved, and the resolution documented in the literature.

Acknowldgements: Not applicable

\section{References}

1. James WP. The epidemiology of obesity: the size of the problem. J Intern Med. 2008;263(6):336-52.

2. Must A, Spadano J, Coakley EH, Field AE, Colditz G, Dietz WH. The disease burden associated with overweight and obesity. JAMA. 1999;282:1523-9.

3. Tambalis KD, Panagiotakos DB, Kavouras SA, Kallistratos AA, Moraiti IP, Douvis SJ, et al. Eleven-year prevalence trends of obesity in Greek children: first evidence that prevalence of obesity is leveling off. Obesity (Silver Spring). 2010;18(1):161-6.

4. Lissau I, Overpeck MD, Ruan WJ et al. Body mass index and overweight in adolescents in 13 European countries, Israel and the United States. Arch Pediatr Adolesc Med. 2004;158: 27-33.

5. World health Organization. (2009). WHO child growth standards: Growth velocity based on weight, length, and head circumference: methods and development. World health Organization. https://apps.who.int/iris/handle/10665/44026.

6. Wang J. Standardization of waist circumference reference data. Am J Clin Nutr. 2006;83:3-4.

7. Rolland-Cachera MF, Brambilla P, Manzoni P, Akrout M, Sironi S, Del Maschio A, Chiumello G. Body composition assessed on the basis of arm circumference and triceps skinfold thickness: A new index validated in children by magnetic resonance imaging. Am J Clin Nutr. 1997;65:1709-13.

8. Cole TJ, Bellizzi MC, Flegal KM, Dietz WH. Establishing a standard definition for child overweight and obesity worldwide: International survey. BMJ. 2000;320:1240-3.

9. Ogden CL, Flegal KM. Changes in terminology for childhood overweight and obesity. Natl Health Stat Report. 2010;25:1-5.

10. Katzmarzyk PT, Srinivasan SR, Chen W, Malina RM, Bouchard C, Berenson GS. Body mass index, waist circumference, and clustering of cardiovascular disease risk factors in a biracial sample of children and adolescents. Pediatrics. 2004;114:198-205.

11. Fox CS, Massaro JM, Hoffmann U, Pou KM, Maurovich-Horvat P, Liu CY, et al. Abdominal visceral and subcutaneous adipose tissue compartments: association with metabolic risk factors in the Framingham Heart Study. Circulation. 2007;116:39-48.

12. Pausova Z, Mahboubi A, Abrahamowicz M, Leonard GT, Perron M, Richer L, et al. Sex differences in the contributions of visceral and total body fat to blood pressure in adolescence. Hypertension. 2012; 59:572-9.

13. Flegal KM, Ogden CL, Yanovski JA, Freedman DS, Shepherd JA, Graubard BI, Borrud LG. High adiposity and high body mass index-for-age in us children and adolescents overall and by race-ethnic group. Am J Clin Nutr. 2010; 91:1020-6. 
14. Laurson KR, Eisenmann JC, Welk GJ. Development of youth percent body fat standards using receiver operating characteristic curves. Am J Prev Med. 2011;41:93-9.

15. Sayer AA, Syddall H, Martin H, Patel H, Baylis D, Cooper C. The developmental origins of sarcopenia. J Nutr Health Aging. 2008;12:427-32.

16. DeFronzo R, Gunnarsson R, Bjorkman M, Olsson M, Wahren J. Effects of insulin on peripheral and splanchnic glucose metabolism in non-insulin-dependent (type II) diabetes mellitus. J Clin Invest. 1985;76:149-55.

17. Steene-Johannessen J, Anderssen SA, Kolle E, Andersen LB. Low muscle fitness is associated with metabolic risk in youth. Med Sci Sports Exerc. 2009;41:1361-7.

18. Bonora E, Targher G. Increased risk of cardiovascular disease and chronic kidney disease in NAFLD. Nat Rev Gastroenterol Hepatol. 2012;9:372-81.

19. Park BS, Yoon JS. Relative skeletal muscle mass is associated with development of metabolic syndrome. Diabetes Metab J. 2013;37:458-64.

20. Ellis KJ, Shypailo RJ, Pratt JA, Pond WG. Accuracy of dual-energy X-ray absorptiometry for bodycomposition measurements in children. Am J Clin Nutr. 1994;60:660-5.

21. Pietrobelli A, Peroni DG, Faith MS. Pediatric body composition in clinical studies: which methods in which situations? Acta Diabetol. 2003;40(1):270-3.

22. Demerath EW, Schubert CM, Maynard LM, Sun SS, Chumlea WC, Pickoff A, Czerwinski SA, Towne B, Siervogel RM. Do changes in body mass index percentile reflect changes in body composition in children? Data from the fels longitudinal study. Pediatrics. 2006;117:487-95.

23. Cole T, Flegal K, Nicholls D, Jackson A. Body mass index cut offs to define thinness in children and adolescents: international survey. BMJ. 2007;335(7612):194-8.

24. Grammatikopoulou MG, Poulimeneas D, Gounitsioti IS, Gerothanasi K, Tsigga M, Kiranas E, The ADONUT Study Group. Prevalence of simple and abdominal obesity in Greek Adolescents: the ADONUT study. Clin Obes. 2014;4:303-8.

25. Tambalis KD, Panagiotakos DB, Psarra G, Sidossis LS. Current data in Greek children indicate decreasing trends of obesity in the transition from childhood to adolescence; results from the National Action for Children's Health (EYZHN) program. J Prev Med Hyg. 2018;59:36-47.

26. Kotanidou EP, Grammatikopoulou MG, Spiliotis BE et al. Ten-year obesity and overweight prevalence in Greek children: a systematic review and meta-analysis of 2001-2010 data. Hormones. 2013;12:537-49.

27. Freedman DS, Khan LK, Serdula MK, Dietz WH, Srinivasan SR, Berenson GS. Relation of age at menarche to race, time period, and anthropometric dimensions: the Bogalusa Heart Study. Pediatrics. 2002;110:e43.

28. Ogden CL, Li Y, Freedman DS, Borrud LG, Flegal KM. Smoothed percentage body fat percentiles for US children and adolescents, 1999-2004. Natl Health Stat Report. 2011;43:1-7. 
29. Nam SY, Kim KR, Cha BS, Song YD, Lim SK, Lee HC, et al. Low-dose growth hormone treatment combined with diet restriction decreases insulin resistance by reducing visceral fat and increasing muscle mass in obese type 2 diabetic patients. Int J Obes Relat Metab Disord. 2001;25:1101-7.

30. Kim K, Hong S, Youg Kim E. Reference values of skeletal muscle mass for Korean children and adolescents using data from the Korean National Health and Nutrition Examination Survey 20092011. Plos One. 2016;11(4):e0153383.

\section{Tables}

Table 1. Anthropometric and body composition measurements in the studied population

\begin{tabular}{|c|c|c|c|c|c|c|c|}
\hline Measurement & $\begin{array}{l}\text { Total } \\
\text { population }\end{array}$ & Males & Females & $\begin{array}{l}\text { p- } \\
\text { value }\end{array}$ & Younger & Older & $\begin{array}{l}\text { p- } \\
\text { value }\end{array}$ \\
\hline Weight (kg) & $44.0 \pm 10.2$ & $44.0 \pm 10.2$ & $44.0 \pm 10.2$ & 0.941 & $41.4 \pm 9.3$ & $46.6 \pm 10.5$ & $<0.001$ \\
\hline Height (cm) & $148.4 \pm 7.6$ & $148.1 \pm 7.3$ & $148.7 \pm 7.8$ & 0.121 & $145.3 \pm 6.7$ & $151.4 \pm 7.1$ & $<0.001$ \\
\hline BMI $\left(\mathrm{kg} / \mathrm{m}^{2}\right)$ & $19.9 \pm 3.5$ & $20.0 \pm 3.5$ & $19.8 \pm 3.5$ & 0.265 & $19.5 \pm 3.4$ & $20.2 \pm 3.6$ & $<0.001$ \\
\hline $\mathrm{FM}(\mathrm{kg})$ & $11.6 \pm 6.7$ & $11.3 \pm 7.0$ & $12.0 \pm 6.4$ & 0.052 & $10.9 \pm 6.3$ & $12.4 \pm 7.1$ & $<0.001$ \\
\hline FM\% & $25.1 \pm 9.9$ & $24.2 \pm 10.3$ & $26.0 \pm 9.4$ & $<0.001$ & $25.0 \pm 10.0$ & $25.2 \pm 9.8$ & 0.669 \\
\hline $\mathrm{FMl}\left(\mathrm{kg} / \mathrm{m}^{2}\right)$ & $5.2 \pm 2.8$ & $5.1 \pm 3.0$ & $5.4 \pm 2.7$ & 0.074 & $5.1 \pm 2.8$ & $5.4 \pm 2.9$ & 0.114 \\
\hline SMM (kg) & $17.3 \pm 3.3$ & $17.7 \pm 3.2$ & $17.1 \pm 3.4$ & 0.001 & $16.2 \pm 2.9$ & $18.5 \pm 3.3$ & $<0.001$ \\
\hline SMM\% & $40.1 \pm 6.4$ & $40.9 \pm 7.0$ & $39.4 \pm 5.6$ & $<0.001$ & $40.0 \pm 6.2$ & $40.4 \pm 6.6$ & 0.160 \\
\hline SMI $\left(\mathrm{kg} / \mathrm{m}^{2}\right)$ & $7.82 \pm 1.05$ & $7.98 \pm 0.97$ & $7.66 \pm 1.11$ & $<0.001$ & $7.64 \pm 1.04$ & $8.00 \pm 1.03$ & $<0.001$ \\
\hline MFR & $2.03 \pm 1.52$ & $2.20 \pm 1.62$ & $1.86 \pm 1.39$ & $<0.001$ & $2.03 \pm 1.50$ & $2.04 \pm 1.54$ & 0.838 \\
\hline
\end{tabular}

Table 1. Anthropometric and body composition measurements (mean \pm standard deviation) in the total population and by gender and age. Statistical significant differences $(p<0.05)$ between males and females and between younger (10-11 years old) and older (11-12 years old) children are shown in bold letters.

Table 2. Anthropometric and body composition measurements in Younger and Older children 


\begin{tabular}{|c|c|c|c|c|c|c|}
\hline & Younger & & & Older & & \\
\hline Measurement & Males & Females & p-value & Males & Females & p-value \\
\hline Weight (kg) & $41.0 \pm 8.6$ & $41.8 \pm 10.0$ & 0.212 & $47.1 \pm 10.9$ & $46.1 \pm 10.1$ & 0.219 \\
\hline Height (cm) & $145.1 \pm 6.0$ & $145.5 \pm 7.4$ & 0.446 & $151.0 \pm 7.4$ & $151.8 \pm 6.9$ & 0.158 \\
\hline BMI $\left(\mathrm{kg} / \mathrm{m}^{2}\right)$ & $19.4 \pm 3.3$ & $19.6 \pm 3.6$ & 0.414 & $20.6 \pm 3.8$ & $19.9 \pm 3.5$ & 0.020 \\
\hline FM (kg) & $10.2 \pm 6.1$ & $11.7 \pm 6.5$ & 0.002 & $12.4 \pm 7.7$ & $12.3 \pm 6.4$ & 0.879 \\
\hline FM\% & $23.5 \pm 10.0$ & $26.5 \pm 9.8$ & $<0.001$ & $24.8 \pm 10.6$ & $25.6 \pm 9.0$ & 0.261 \\
\hline $\mathrm{FMI}\left(\mathrm{kg} / \mathrm{m}^{2}\right)$ & $4.8 \pm 2.8$ & $5.4 \pm 2.8$ & 0.003 & $5.4 \pm 3.2$ & $5.3 \pm 2.6$ & 0.673 \\
\hline SMM (kg) & $16.5 \pm 2.5$ & $15.9 \pm 3.3$ & 0.016 & $18.7 \pm 3.5$ & $18.2 \pm 3.2$ & 0.018 \\
\hline SMM\% & $41.0 \pm 6.2$ & $38.7 \pm 6.0$ & $<0.001$ & $40.8 \pm 7.8$ & $40.0 \pm 5.2$ & 0.094 \\
\hline $\mathrm{SMI}\left(\mathrm{kg} / \mathrm{m}^{2}\right)$ & $7.79 \pm 0.90$ & $7.47 \pm 1.15$ & $<0.001$ & $8.16 \pm 1.00$ & $7.84 \pm 1.04$ & $<0.001$ \\
\hline MFR & $2.23 \pm 1.65$ & $1.80 \pm 1.30$ & $<0.001$ & $2.16 \pm 1.60$ & $1.92 \pm 1.47$ & 0.029 \\
\hline
\end{tabular}

Table 2. Anthropometric and body composition measurements (mean \pm standard deviation) in younger (10-11 years old) and older (11-12 years old) children by gender. Statistical significant differences $(p<0.05)$ between Males and Females are shown in bold letters.

Table 3. Anthropometric and body composition measurements in Male and Female children

\begin{tabular}{|lllllll|}
\hline & Males & \multicolumn{5}{c|}{ Females } \\
\hline Measurement & Younger & Older & p-value & Younger & Older & p-value \\
\hline Weight $(\mathrm{kg})$ & $41.0 \pm 8.6$ & $47.1 \pm 10.9$ & $<0.001$ & $41.8 \pm 10.0$ & $46.1 \pm 10.1$ & $<0.001$ \\
\hline Height $(\mathrm{cm})$ & $145.1 \pm 6.0$ & $151.0 \pm 7.4$ & $<0.001$ & $145.5 \pm 7.4$ & $151.8 \pm 6.9$ & $<0.001$ \\
\hline BMI $\left(\mathrm{kg} / \mathrm{m}^{2}\right)$ & $19.4 \pm 3.3$ & $20.6 \pm 3.8$ & $<0.001$ & $19.6 \pm 3.6$ & $19.9 \pm 3.5$ & 0.267 \\
\hline FM $(\mathrm{kg})$ & $10.2 \pm 6.1$ & $12.4 \pm 7.7$ & $<0.001$ & $11.7 \pm 6.5$ & $12.3 \pm 6.4$ & 0.178 \\
\hline FM\% & $23.5 \pm 10.0$ & $24.7 \pm 10.6$ & 0.104 & $26.5 \pm 9.8$ & $25.6 \pm 9.0$ & 0.216 \\
\hline FMI $\left(\mathrm{kg} / \mathrm{m}^{2}\right)$ & $4.8 \pm 2.8$ & $\mathbf{5 . 4 \pm 3 . 2}$ & $\mathbf{0 . 0 0 8}$ & $5.4 \pm 2.8$ & $5.3 \pm 2.6$ & 0.515 \\
\hline SMM $(\mathrm{kg})$ & $16.5 \pm 2.5$ & $18.7 \pm 3.5$ & $<0.001$ & $15.9 \pm 3.3$ & $18.2 \pm 3.2$ & $<0.001$ \\
\hline SMM\% & $41.0 \pm 6.2$ & $40.8 \pm 7.8$ & 0.679 & $38.7 \pm 6.0$ & $39.9 \pm 5.1$ & 0.004 \\
\hline SMI $\left(\mathrm{kg} / \mathrm{m}^{2}\right)$ & $7.79 \pm 0.90$ & $\mathbf{8 . 1 6} \pm 1.00$ & $<0.001$ & $7.47 \pm 1.15$ & $7.84 \pm 1.04$ & $<0.001$ \\
\hline MFR & $2.23 \pm 1.65$ & $2.16 \pm 1.60$ & 0.560 & $1.80 \pm 1.30$ & $1.92 \pm 1.47$ & 0.276 \\
\hline
\end{tabular}


Table 3. Anthropometric and body composition measurements (mean \pm standard deviation) in male and female children by age (Younger: 10-11 years old and Older: 11-12 years old). Statistical significant differences $(p<0.05)$ between males and females are shown in bold letters.

Table 4. Differences in the studied indexes among BMI\% categories 


\begin{tabular}{|c|c|c|c|c|c|c|}
\hline \multirow[b]{2}{*}{ Measurement } & \multicolumn{3}{|c|}{ BMI percentage } & \multicolumn{3}{|l|}{ P-value } \\
\hline & $<85$ & $85-95$ & $>95$ & $\begin{array}{l}<85 \text { vs. } \\
85-95\end{array}$ & $\begin{array}{l}<85 \text { vs. } \\
>95\end{array}$ & $\begin{array}{l}85-95 \text { vs. } \\
>95\end{array}$ \\
\hline FM Total population (kg) & $7.8 \pm 3.5$ & $14.9 \pm 3.7$ & $23.4 \pm 6.7$ & $<0.001$ & $<0.001$ & $<0.001$ \\
\hline FM Boys (kg) & $7.2 \pm 0.1$ & $14.8 \pm 0.2$ & $23.6 \pm 0.4$ & $<0.001$ & $<0.001$ & $<0.001$ \\
\hline FM Girls (kg) & $8.4 \pm 0.2$ & $15.0 \pm 0.2$ & $23.1 \pm 0.4$ & $<0.001$ & $<0.001$ & $<0.001$ \\
\hline FM Younger (kg) & $7.3 \pm 3.2$ & $14.2 \pm 3.7$ & $22.0 \pm 6.0$ & $<0.001$ & $<0.001$ & $<0.001$ \\
\hline FM Older (kg) & $8.4 \pm 3.7$ & $15.6 \pm 3.5$ & $24.5 \pm 7.2$ & $<0.001$ & $<0.001$ & $<0.001$ \\
\hline FM\% Total population & $20.0 \pm 7.5$ & $30.4 \pm 6.9$ & $38.5 \pm 7.1$ & $<0.001$ & $<0.001$ & $<0.001$ \\
\hline FM\% Boys & $18.5 \pm 0.3$ & $30.3 \pm 0.5$ & $38.9 \pm 0.7$ & $<0.001$ & $<0.001$ & $<0.001$ \\
\hline FM\% Girls & $21.7 \pm 0.3$ & $30.5 \pm 0.5$ & $37.9 \pm 0.8$ & $<0.001$ & $<0.001$ & $<0.001$ \\
\hline FM\% Younger & $19.8 \pm 7.3$ & $30.9 \pm 7.5$ & $38.4 \pm 6.7$ & $<0.001$ & $<0.001$ & $<0.001$ \\
\hline FM\% Older & $20.3 \pm 7.7$ & $29.9 \pm 6.1$ & $38.5 \pm 7.5$ & $<0.001$ & $<0.001$ & $<0.001$ \\
\hline $\begin{array}{l}\text { FMI Total population } \\
\left(\mathrm{kg} / \mathrm{m}^{2}\right)\end{array}$ & $3.6 \pm 1.5$ & $6.7 \pm 1.6$ & $10.2 \pm 2.6$ & $<0.001$ & $<0.001$ & $<0.001$ \\
\hline FMI Boys $\left(\mathrm{kg} / \mathrm{m}^{2}\right)$ & $3.4 \pm 0.1$ & $6.6 \pm 0.1$ & $10.4 \pm 0.2$ & $<0.001$ & $<0.001$ & $<0.001$ \\
\hline FMI Girls $\left(\mathrm{kg} / \mathrm{m}^{2}\right)$ & $3.9 \pm 0.1$ & $6.7 \pm 0.1$ & $10.0 \pm 0.2$ & $<0.001$ & $<0.001$ & $<0.001$ \\
\hline FMI Younger $\left(\mathrm{kg} / \mathrm{m}^{2}\right)$ & $3.5 \pm 1.5$ & $6.6 \pm 1.7$ & $10.0 \pm 2.4$ & $<0.001$ & $<0.001$ & $<0.001$ \\
\hline FMI Older $\left(\mathrm{kg} / \mathrm{m}^{2}\right)$ & $3.7 \pm 1.6$ & $6.7 \pm 1.6$ & $10.4 \pm 2.7$ & $<0.001$ & $<0.001$ & $<0.001$ \\
\hline $\begin{array}{l}\text { SMM Total population } \\
\text { (kg) }\end{array}$ & $16.3 \pm 3.0$ & $18.6 \pm 3.1$ & $19.6 \pm 3.3$ & $<0.001$ & $<0.001$ & 0.001 \\
\hline SMM Boys (kg) & $16.7 \pm 0.1$ & $18.9 \pm 0.2$ & $19.5 \pm 0.3$ & $<0.001$ & $<0.001$ & 0.332 \\
\hline SMM Girls (kg) & $15.9 \pm 0.1$ & $18.4 \pm 0.2$ & $19.8 \pm 0.3$ & $<0.001$ & $<0.001$ & 0.001 \\
\hline SMM Younger (kg) & $15.2 \pm 2.5$ & $17.3 \pm 2.8$ & $18.6 \pm 3.0$ & $<0.001$ & $<0.001$ & $<0.001$ \\
\hline SMM Older (kg) & $17.4 \pm 3.1$ & $19.9 \pm 2.9$ & $20.5 \pm 3.4$ & $<0.001$ & $<0.001$ & 0.355 \\
\hline SMM\% Total population & $42.5 \pm 5.3$ & $37.9 \pm 6.4$ & $32.8 \pm 3.9$ & $<0.001$ & $<0.001$ & $<0.001$ \\
\hline SMM\% Boys & $43.5 \pm 0.3$ & $38.7 \pm 0.4$ & $32.6 \pm 0.6$ & $<0.001$ & $<0.001$ & $<0.001$ \\
\hline SMM\% Girls & $41.5 \pm 0.2$ & $37.2 \pm 0.3$ & $33.1 \pm 0.5$ & $<0.001$ & $<0.001$ & $<0.001$ \\
\hline SMM\% Younger & $42.2 \pm 4.7$ & $37.6 \pm 6.9$ & $32.8 \pm 3.5$ & $<0.001$ & $<0.001$ & $<0.001$ \\
\hline SMM\% Older & $42.8 \pm 5.8$ & $38.4 \pm 5.7$ & $32.7 \pm 4.2$ & $<0.001$ & $<0.001$ & $<0.001$ \\
\hline
\end{tabular}




\begin{tabular}{|lllllll|}
$\begin{array}{l}\text { SMI Total population } \\
\left(\mathrm{kg} / \mathrm{m}^{2}\right)\end{array}$ & $7.48 \pm 0.93$ & $8.28 \pm 1.02$ & $8.58 \pm 0.94$ & $<0.001$ & $<0.001$ & 0.002 \\
\hline SMI Boys $\left(\mathrm{kg} / \mathrm{m}^{2}\right)$ & $7.68 \pm 0.04$ & $8.40 \pm 0.07$ & $8.57 \pm 0.09$ & $<0.001$ & $<0.001$ & 0.343 \\
\hline SMI Girls $\left(\mathrm{kg} / \mathrm{m}^{2}\right)$ & $7.25 \pm 0.05$ & $8.16 \pm 0.07$ & $8.58 \pm 0.11$ & $<0.001$ & $<0.001$ & 0.004 \\
\hline SMI Younger $\left(\mathrm{kg} / \mathrm{m}^{2}\right)$ & $7.31 \pm 0.88$ & $8.04 \pm 1.12$ & $8.45 \pm 0.87$ & $<0.001$ & $<0.001$ & 0.002 \\
\hline SMI Older $\left(\mathrm{kg} / \mathrm{m}^{2}\right)$ & $7.64 \pm 0.94$ & $8.51 \pm 0.84$ & $8.68 \pm 0.99$ & $<0.001$ & $<0.001$ & 0.400 \\
\hline MFR Total population & $2.54 \pm 1.63$ & $1.38 \pm 1.00$ & $0.91 \pm 0.32$ & $<0.001$ & $<0.001$ & $<0.001$ \\
\hline MFR Boys & $2.82 \pm 0.07$ & $1.33 \pm 0.10$ & $0.90 \pm 0.14$ & $<0.001$ & $<0.001$ & 0.039 \\
\hline MFR Girls & $2.24 \pm 0.06$ & $1.43 \pm 0.09$ & $0.92 \pm 0.14$ & $<0.001$ & $<0.001$ & 0.009 \\
\hline MFR Younger & $2.54 \pm 1.63$ & $1.34 \pm 0.80$ & $0.90 \pm 0.26$ & $<0.001$ & $<0.001$ & 0.039 \\
\hline MFR Older & $2.54 \pm 1.62$ & $1.43 \pm 1.15$ & $0.92 \pm 0.37$ & $<0.001$ & $<0.001$ & 0.010 \\
\hline
\end{tabular}

Table 4. Anthropometric and body composition measurements (mean \pm standard deviation) in the total population, males, females, younger (10-11 years old) and older (11-12 years old) children by BMI\%. Statistical significant differences $(p<0.05)$ are shown in bold letters.

\section{Figures}



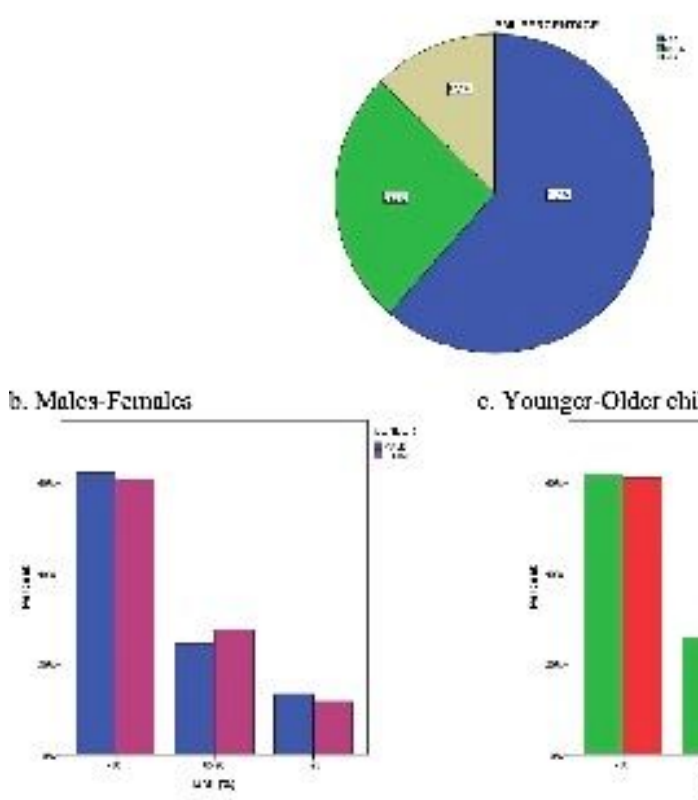

c. Younger-Older children

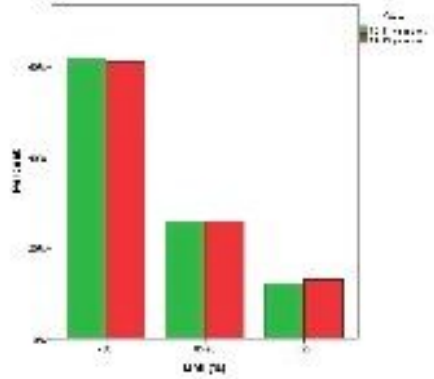

d. Muley-rinules (Youngar)

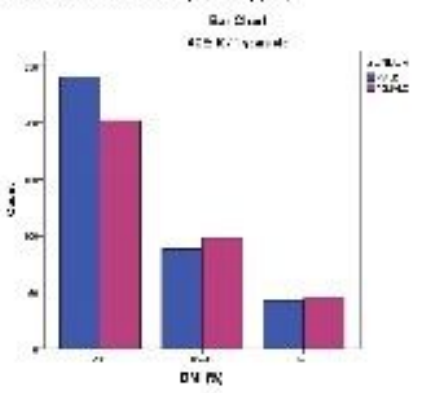

c. Malky-Fimules (Older)

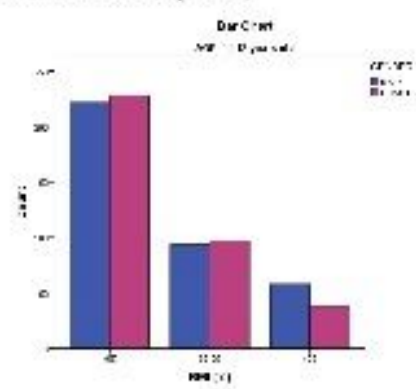

f. Yotmeger-Ulder (Males) g. Yourger-Olvikr (limales)
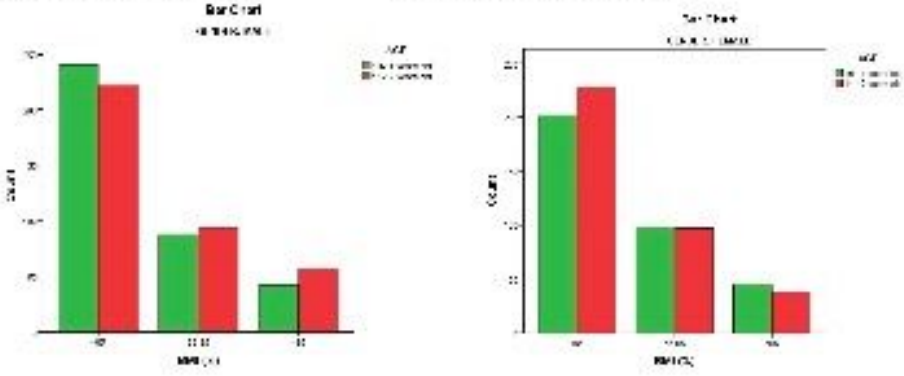

\section{Figure 1}

BMI distribution in the studied population. BMI\% distribution in: a) Total population, b) Males and females, c) Younger and older children, d) Younger males and females, e) Older males and females, f) Younger and older males, g) Younger and older females. In all comparisons with respect to BMI\% distribution, no statistically significant difference exists (Pearson chi-square test, $p>0.05$ ). 